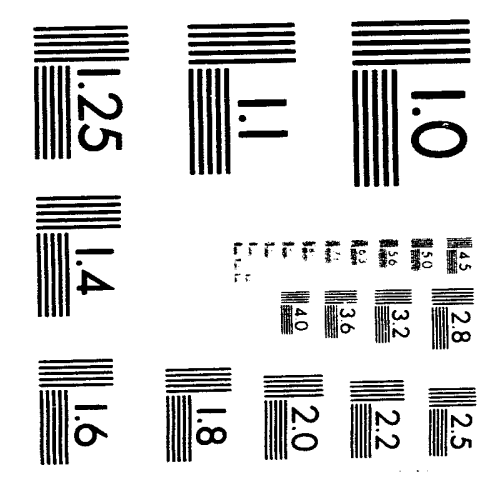



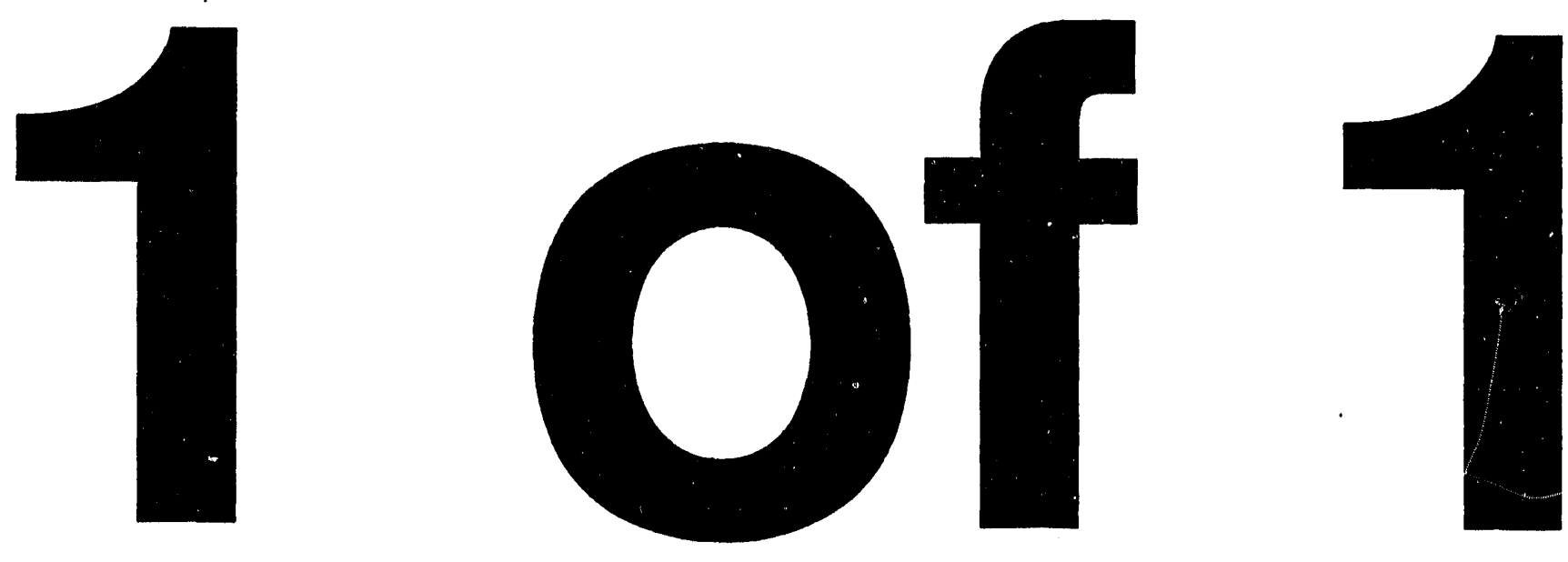

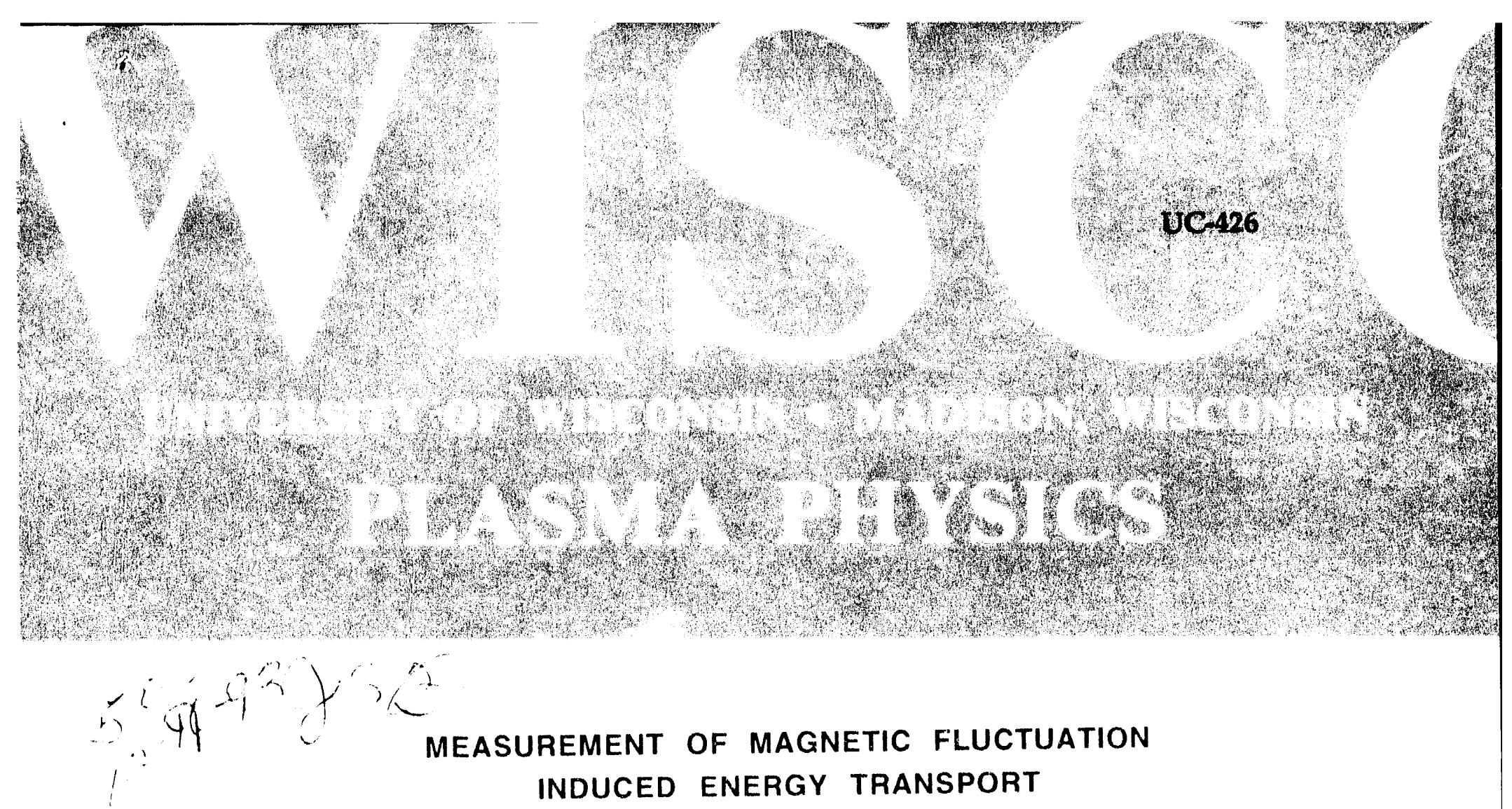

G. Fiksel, S.C. Prager, W. Shena and M. Stoneking

DOE/ER/53198-227

November 1993

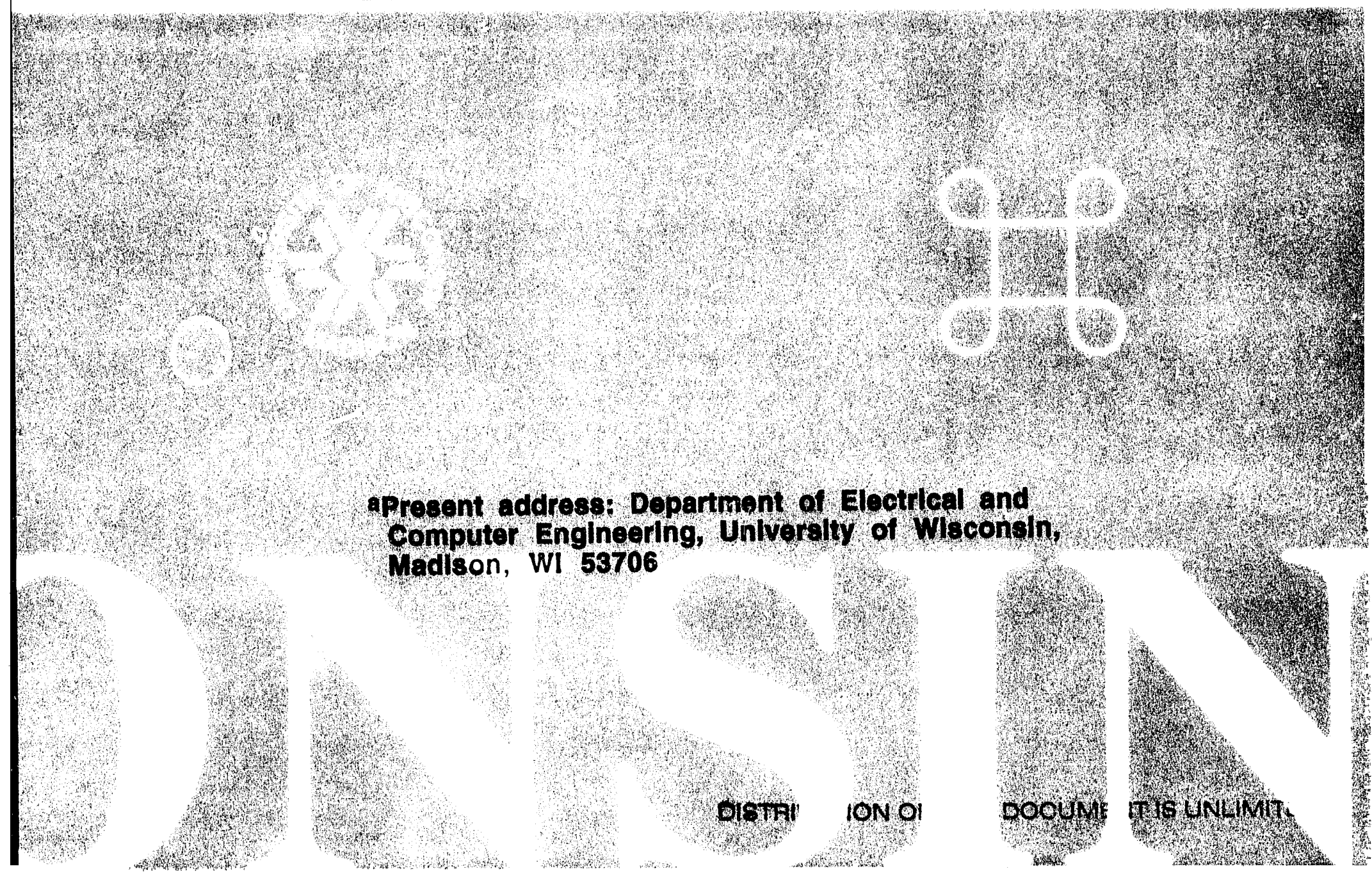




\section{NOTICE}

This report was prepared as an account of work sponsored by an agency of the United States Government. Neither the United States nor any agency thereof, nor any of their employees, makes any warranty, expressed or implied, or assumes any legal liability or responsibility for any third party's use or the results of such use of any information, apparatus, product or process disclosed in this report, or represents that its use by such third party would not infringe privately owned rights.

Printed in the United States of America Available from

National Technical Information Service

U.S. Department of Commerce

5285 Port Royal Road

Springfield, VA 22161

NTIS Price codes

Printed copy: $\quad$ A02

Microfiche copy: A01 


\title{
Measurement of Magnetic Fluctuation Induced Energy Transport
}

\author{
G. Fiksel, S.C. Prager, W. Shena, and M. Stoneking \\ Department of Physics \\ University of $W$ isconsin \\ Madison, Wisconsin 53706
}

\begin{abstract}
The local electron energy flux produced by magnetic fluctuations has been measured directly in the MST reversed field pinch (overthe radial range r/a>0.75). The flux, produced by electrons traveling parallel to a fluctuating magnetic field, is obtained from correlation between the fluctuations in the parallel heat flux and the radial magnetic field. The fluctuation induced flux is la rge $\left(100 \mathrm{~kW} / \mathrm{cm}^{2}\right)$ in the "core" $(\mathrm{r} / a<0.85)$ and small ( $<10-30 \mathrm{~kW} / \mathrm{cm}^{2}$ ) in the edge.
\end{abstract}

It has long been recognized that fluctuations in the magnetic field is a potent mechanism for the anomalous transport of energy in confined plasmas. The energy transport process originates from the plasma particles motion along the magnetic field, which can contain fluctuations in the radial direction (perpendicular to the confining equilibrium magnetic surfaces). A key feature is that the transport can be large even if the fluctuation amplitude is extremely small. If the fluctuations are resonant with the equilibrium magnetic field (i.e., the fluctuation amplitude is constant along an equilibrium field line) then a small fluctuation can introduce stochasticity to the field line trajectories. Particles following the chaotically wandering field lines can rapidly carry energy across the plasma. 1,2

\footnotetext{
a Present address: Department of Electrical and Computer Engineering, University of Wisconsin, Madison, WI 53706.
} 
After several decades of research it remains an open question whether magnetic fluctuations are responsible for anomalous transport in confinement systems, such as the tokamak, stellarator, and reversed field pinch. In all these devices, magnetic fluctuations have been measured and anomalous energy transport is observed. However, causality between the two has never been proved or disproved.

In this Letter we report direct measurement of the electron energy flux specifically generated by the magnetic fluctuations in the MST reversed field pinch (RFP). The measurement was performed in the outer $25 \%$ of the minor radius $(r / a>0.75)$. We establish that magnetic fluctuation induced energy transport is large in the "core" $(r / a<0.85)$ and small in the extreme edge. To accomplish this measurement a novel diagnostic has been developed which can similarly be applied to the edge of other configurations.

Fluctuation induced transport fluxes are given by quadratic correlations of appropriate fluctuating quantities. The radial energy flux arising from electron motion parallel to the magnetic field is given by $Q_{r}=Q_{\|}$. $\hat{r}=(Q \cdot \hat{b})(\hat{b} \cdot \hat{r})$ where $\hat{b}$ and $\hat{r}$ are unit vectors along the magnetic field and the radial direction respectively. Separating $Q$ and $\hat{b}$ into equilibrium and fluctuating quantities yields the ensemble-averaged radial energy flux ${ }^{3}$

$$
\mathrm{Q}_{\mathrm{r}}=\frac{\left\langle\tilde{\mathrm{Q}}_{\|} \tilde{\mathrm{B}}_{\mathrm{r}}>\right.}{\mathrm{B}}
$$

where $\tilde{Q}_{\|}$is the fluctuating electron heat flux parallel to the equilibrium magnetic field (i.e., $\left.\tilde{Q}_{\|}=\int_{v} \|\left(\mathrm{mv}^{2} / 2\right) \tilde{\mathrm{f}}(\mathrm{v}) \mathrm{dv}\right), \quad \tilde{\mathrm{B}}_{\mathrm{r}}$ is the fluctuating radial magnetic field, and $B$ is the equilibrium field. The ensemble average $<>$ is realized experimentally by averaging many time records. Since the phase of 
the fluctuations is random over a magnetic surface, the ensemble average approximates a magnetic surface average. A second term enters in the above expression $\left(Q_{\|}<\widetilde{B}_{\|} \tilde{B}_{r}>/ B^{2}\right)$, but is omitted since it is measured to be small in MST. We also note that magnetic fluctuations can contribute to energy transport through the $\mathrm{E} \times \mathrm{B}$ drift that results from the fluctuating inductive electric field that accompanies the magnetic field. This inechanism is expected to be small in MST and is not considered here. Transport from fluctuating electrostatic fields has been extensively studied with Langmuir probes in the edge of many devices. The key to measuring the energy flux from fluctuating magnetic field is to obtain $\quad \tilde{Q}_{\|}$locally within the plasma. To this end, we have developed a fast, insertable bolometer. 4

$\mathrm{MST}^{5}$ is a relatively large $\mathrm{RFP}(\mathrm{a}=0.5 \mathrm{~m}, \mathrm{R}=1.5 \mathrm{~m})$ with moderate plasma current $(\mathrm{I}<0.7 \mathrm{MA})$. To permit diagnostic insertion into the plasma, the experiments reported here were operated at reduced parameters. For measurements at the plasma edge $(r / a>0.9)$ the plasma current was maintained at $220 \mathrm{kA}$; for deeper insertion $(0.75<\mathrm{r} / \mathrm{a}<0.9)$ the current was reduced to $120 \mathrm{kA}$. The line-averaged density was $0.8-1 \times 10^{13}$ $\mathrm{cm}^{-3}$, and the central electron temperature is $100-150 \mathrm{eV}$. The confinement properties and fluctuation characteristics are relatively unchanging over the full current range of MST, so that we believe that the present results are not peculiar to low current. Magnetic fluctuations have been studied extensively in MST.6,7,3 Nearly all the fluctuation power ( $>90 \%$ ) resides in several modes at low frequency ( $\mathrm{f}<30 \mathrm{kHz}$ ) with poloidal and toroidal mode number $m=1$ and $n=5-8$. Detailed comparison between the nonlinear MHD computation and experimental measurements, including details of nonlinear coupling, 8 has established that the fluctuations are nonlinearly coupled, global, tearing modes resonant in the core. These modes are capable of 
breaking the magnetic surfaces within the reversal surface $(a t r / a \approx 0.85)$. The higher frequency fluctuations (f $>50 \mathrm{kHz}$ ) are small scale turbulence resonant with the local magnetic field. Their origin is not yet established.

The parallel electron heat flux was measured with a fast pyrobolometer, ${ }^{4}$ capable of detecting the fluctuations, $\tilde{Q}_{\| 1}$. The schematic of the bolometer is shown in Fig. 1. The heart of the bolometer is a pyrocrystal of $\mathrm{LiNbO}_{3}$. The diameter of the crystal is $1 \mathrm{~cm}$ and the thickness is $1 \mathrm{~mm}$. When exposed to a plasma (or any other source of thermal energy) the crystal generates electric current that is proportional to the absorbed power. The signal is measured with a fast current-to-voltage converter. The pyrocrystal itself was absolutely calibrated and the frequency bandwidth was measured to be $150 \mathrm{kHz}$. Details of calibration and the design are found in Ref. 3. Both sides of the crystal are metalized and the side exposed to the plasma is grounded. The main source of the heat flux at the plasma edge is electrons streaming along the ragnetic field lines. The electrons enter the bolometer through a small cperture (1 $\mathrm{mm}$ in diameter and $1 \mathrm{~mm}$ in length) in the protective boron nitride shroud. The bolometer is two-sided; therefore, when aligned along magnetic lines it measures the field aligned heat flux simultaneously in two opposite directions, which yields the net flux.

With the biased repelling electrode we could either detect the plasma electron energy distribution function or gate the electron flux. The gating decreases the total energy deposited into the bolometer, so the bolometer can be inserted deeper into the plasma without damage. In the experiments described below the bolometer is gated with a duration of $2 \mathrm{~ms}$ during the plasma current flattop. The geometric transparency of the entrance aperture was evaluated by a Monte-Carlo simulation of the incoming electron flux in the magnetic field, using the measured electron distribution function 9 . The 
calculated transparency is about $50 \%$, in agreement with the value inferred from other bolometric measurements in MST 9 .

The fluctuation of the magnetic field was measured by a miniature magnetic coil. The poloidal separation of the bolometer and the magnetic probe was $10 \mathrm{~cm}$ which is well within the poloidal correlation length of $\sim 0.5$ $1 \mathrm{~m}$ for the dominant $\mathrm{m}=1$ tearing modes in the MST. The radial separation of the two probes was kept within $\pm 0.5 \mathrm{~cm}$.

The parallel heat flux measured by the bolometer is shown in Fig. 2, for the forward (parallel to the electron drift) direction. The equilibrium component of the heat flux has a large anisotropy, with the heat flux in the electron drift direction dominating by a factor of 5 to 10 (Fig. 3). Measurements with an electron energy analyzer (EEA) ${ }^{9}$ reveal that in MST, as in other RFPs, 10 the current at the cold edge is dominated by fast electrons which can be described as a drifted bi-Maxwellian with $\mathrm{T}_{\|} \approx 80-115 \mathrm{eV}$ and $\mathrm{T}_{\perp}$ $\approx 20-30 \mathrm{eV}$ and $\mathrm{E}_{\mathrm{drift}}=\mathrm{mv}^{2} \mathrm{drift} / 2 \approx 10-20 \mathrm{eV}$. These particle flux measurements are consistent with the pyrobolometer heat flux measurements, if we relate the heat flux and particle flux as $Q_{\|}=(j \| / e)\left(3 / 2 T_{\|}+\right.$ $\mathrm{T}_{\perp}+\mathrm{E}_{\mathrm{drift}}$ ). For the measured $\mathrm{Q} \|=2000 \mathrm{~W} / \mathrm{cm}^{2}$ we find that the fast electron current density $\mathrm{j} \|=10 \mathrm{~A} / \mathrm{cm}^{2}$, roughly $75 \%$ to $100 \%$ of the total current density. The fast electron temperature inferred from a repeller voltage scan of the pyrobolometer also agrees with EEA measurements. An additional salient feature of the parallel heat flux is the large bursts, which coincide with the crash of sawtooth oscillations.

The fluctuations in the parallel heat flux, $\tilde{Q}_{\|}$, are large, roughly $50 \%$, as is evident from Fig. 2. The fluctuation amplitude increases with radius, as seen in Fig. 3. The frequency spectra of $\widetilde{Q}_{\|}$and $\widetilde{B}_{r}$ are similar (Fig. 4a), with both peaking at low frequency. The peak at the extreme low end of the 
bandwidth represents the sawtooth oscillations. The gentle peak apparent at about $10 \mathrm{kHz}$ in both signals arises from the tearing oscillations.

To determine the radial energy flux driven by these fluctuations (eq 1) we have evaluated the cross-coherence between $\tilde{Q}_{\|}$and $\tilde{B}_{\mathfrak{r}}$ from an ensembles of $60-120$ reproducible discharges. The magnitude and phase of the cross-coherence is displayed in Figs $4 \mathrm{~b}$ and $4 \mathrm{c}$ (for $\mathrm{r} / \mathrm{a}=0.75$ ). The coherence is strong and restricted to low frequency $(\approx 10 \mathrm{kHz})$. This coherence arises from the several dominant tearing fluctuations. The coherence decreases with radius, reaching $\approx 0.2$ at $\mathrm{r} / \mathrm{a}=0.85$ (reversal surface) and $=0.3$ near at $r / a=0.95$. The cross-power frequency spectrum is displayed in Fig. $4 \mathrm{~d}$. It is clear that the energy flux from magnetic fluctuations arises mainly from the low frequency tearing oscillations. The higher frequency microturbulence has a weak cross-coherence and low amplitude; hence, it does not contribute significantly to the energy flux from magnetic fluctuations. The cross-phase at the frequency of maximum coherence is about 60 degrees, not optimal for transport. However, the resulting energy flux can be large since the fluctuation amplitudes are large.

From the coherence measurements the radial energy flux, $\mathrm{Q}_{\mathrm{r}}=$ $<\widetilde{\mathrm{Q}}_{\|} \widetilde{\mathrm{B}}_{\mathrm{r}}>/ \mathrm{B}$, is calculated. The radial profile of the energy transport is shown in Fig. 5. In order to compare it with the total energy flux we also show the magnetic energy flux relative to the total Ohmic power normalized to the magnetic surface area at the corresponding radius Precise determination of the radial dependence of $t: 2$ total energy flux requires profile information not available. We see that at the plasma edge the heat transport from magnetic fluctuations is low, but inside the reversal surface $(r / a<0.85)$ it constitutes a significant fraction of the total flux. The observed radial dependence of the anomalous transport is consistent with expectation of field line stochasticity. 
It is anticipated from MHD computation that the magnetic surfaces within the reversal surface are broken by the dominant magnetic fluctuations resonant in the core. The edge magnetic surfaces are not similarly disturbed since the tearing fluctuations are not resonant in that region.

We conclude with four points, two of which are general and two focused on the RFP. First, we have definetely, by measuring the local $\tilde{Q}_{\|}$, and $\tilde{\mathrm{B}}_{\mathrm{r}}$, and their correlation, demonstrated that magnetic fluctuations can drive significant energy transport. This demonstration is definitive since we have directly measured the energy flux driven by magnetic fluctuations. The radial profile and frequency dependence of the fluctuation induced flux is in accord with the expectation that internally resonant tearing oscillations are responsible for the transport. In the extreme edge, where the tearing oscillations do not break magnetic surfaces, the energy flux from magnetic fluctuations is small. Second, the technique, based on the development of a fast, insertable bolometer, can be applied to the edge of other experiments, such as tokamaks and stellarators, to similarly discover the contribution of magnetic fluctuations to transport. Third, the cause of anomalous energy transport in the extreme edge of the RFP remains unknown. Earlier work $11,12,13$ indicates that electrostatic fluctuations, operating on the bulk of the electron velocity distribution function, are not responsible. In MST we will be exploring the contribution of electrostatic transport of fast electrons.

Finally, with the presents results, the RFP concept might be in the unique position of being accompanied by a basic understanding of the origin of both the dominant fluctuations and the anomalous energy transport. This feature is subject to the caveat that the results reported here were limited to one device under restricted conditions. Nonetheless, a physics basis now 
exists for the control of fluctuations and transport in the RFP, and appropriate techniques are presently being formulated.

The authors appreciate discussions with many MST group members.

This work has been supported by the US. Department of Energy. 


\section{REFERENCES}

1. J.D. Callen, Phys Rev. Lett., 39, 1540 (1977).

2 A.B. Rechester and M.N.Rosenbluth, Phys. Rev. lett.40, 38 (1978).

3 S.C. Prager, Plasma Phys. Controlled Fusion, 32, 903 (1990).

4. G. Fiksel, D. Holly, and J. Frank. Rev. Sci. Instrum, to be published in1993.

5. R.N. Dexter et al., Fusion Technology 19,131 (1991).

6. A.F. Almagri et al., Phys. Fluids B 4, 4080 (1992).

7. J.S. Sarff et al., Phys. Fluids B 52540 (1993).

8. S. Assadi, S.C. Prager, and K.L. Sidikman, Phys. Rev. Lett. 69, 281 (1992).

9. M. Stoneking et al., will be published in Bull. Amer. Phys. Soc. 38, 4S2, (1993)

10. J.C. Ingraham et al., Fluids B 2, 143 (1990).

11. H. Ji et al., Phys. Rev. Lett. 67, 62 (1991).

12. T.D. Rempel et al., Fluids B 4, 2136 (1992)

13. H.Y.W. Tsui Nucl. Fusion 28, 1543 (1998) 


\section{FIGURE CAPTIONS}

Figure 1. Schematic of the pyrobolometer.

Figure 2. Parallel heat flux $Q_{\|}$at $4 \mathrm{~cm}$ from the wall $(\mathrm{r} / \mathrm{a}=.92)$.

Figure 3. Radial distribution of parallel heat flux and its fluctuation. The forward direction corresponds to the direction of fast electrons drift.

Figure 4. Frequency spectra of a) fluctuation of $Q_{\|}$and $\left.B_{r} ; b\right)$ cross coherence of $Q_{\|}$and $\left.B_{r}, c\right)$ cross phase, and d) cross-power

Figure 5. Radial profile of magnetic fluctuation induced heat transport $\mathrm{Q}_{\mathrm{r}}=\left\langle\tilde{\mathrm{Q}}_{\|} \tilde{\mathrm{B}}_{\mathrm{r}}\right\rangle / \mathrm{B}$. The dashed line shows the fluctuation heat transport relative to the Ohmic power POhmic normalized to the magnetic surface area at the corresponding radius. 


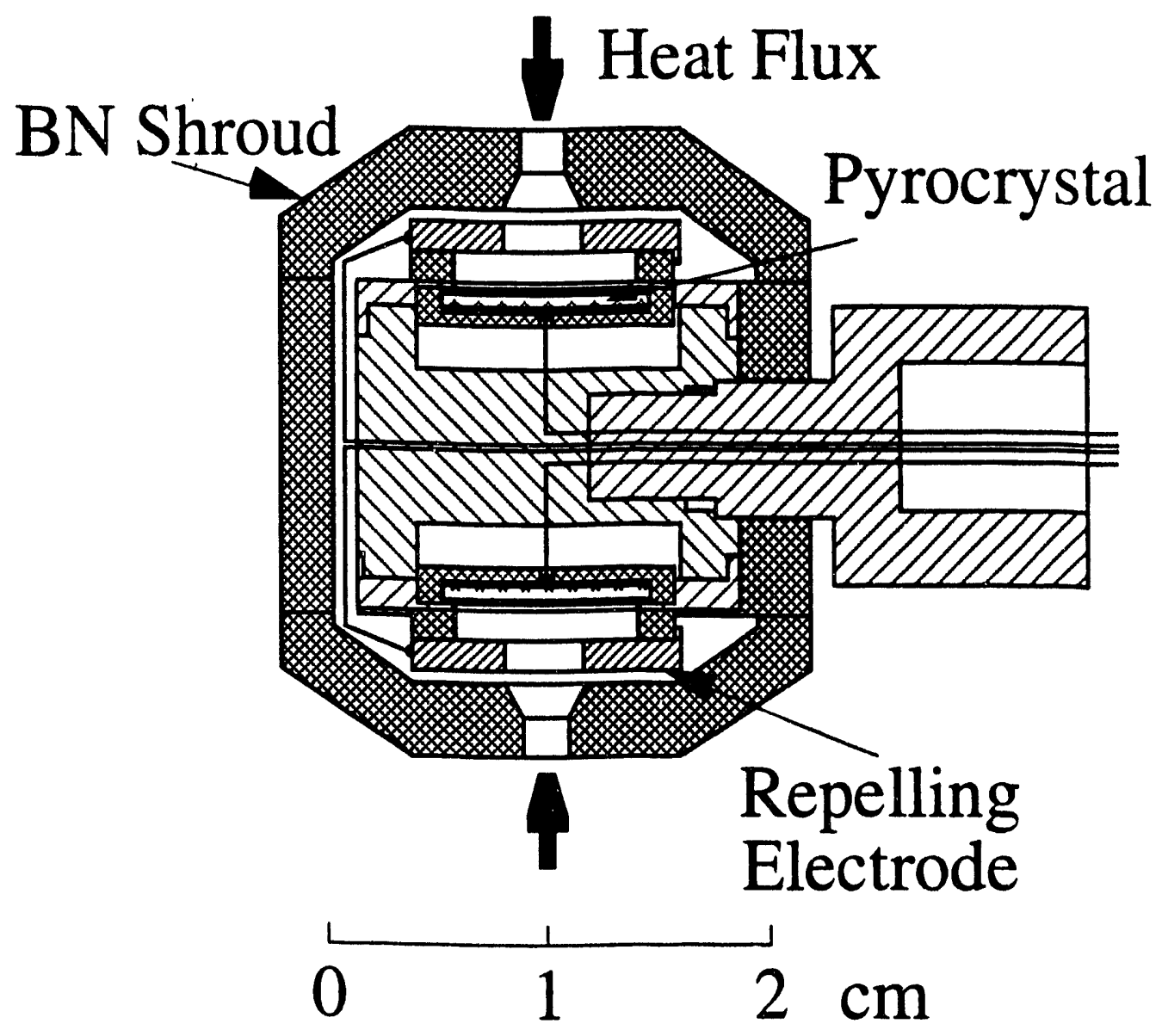

Fig. 1 


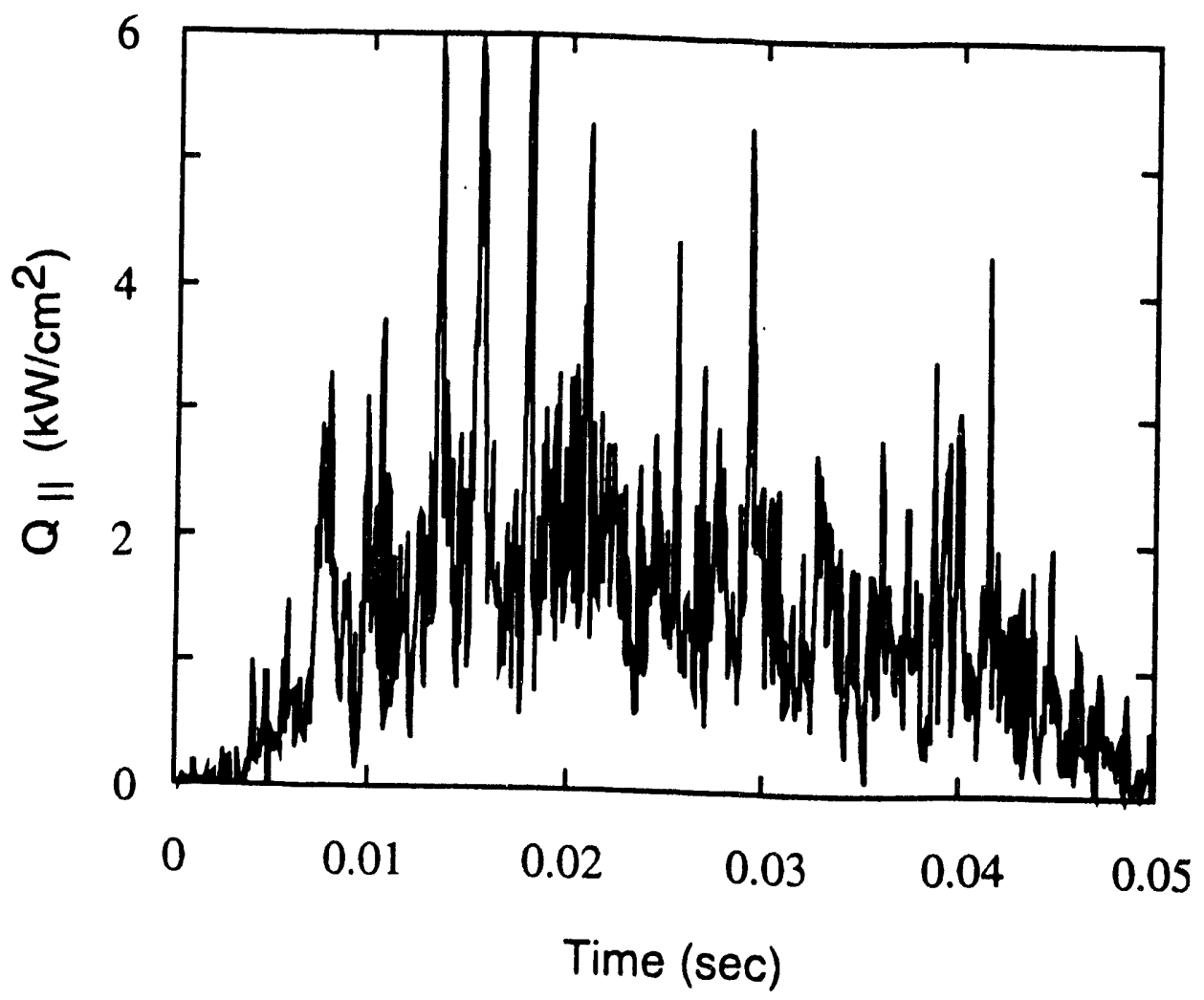

Fig. 2 


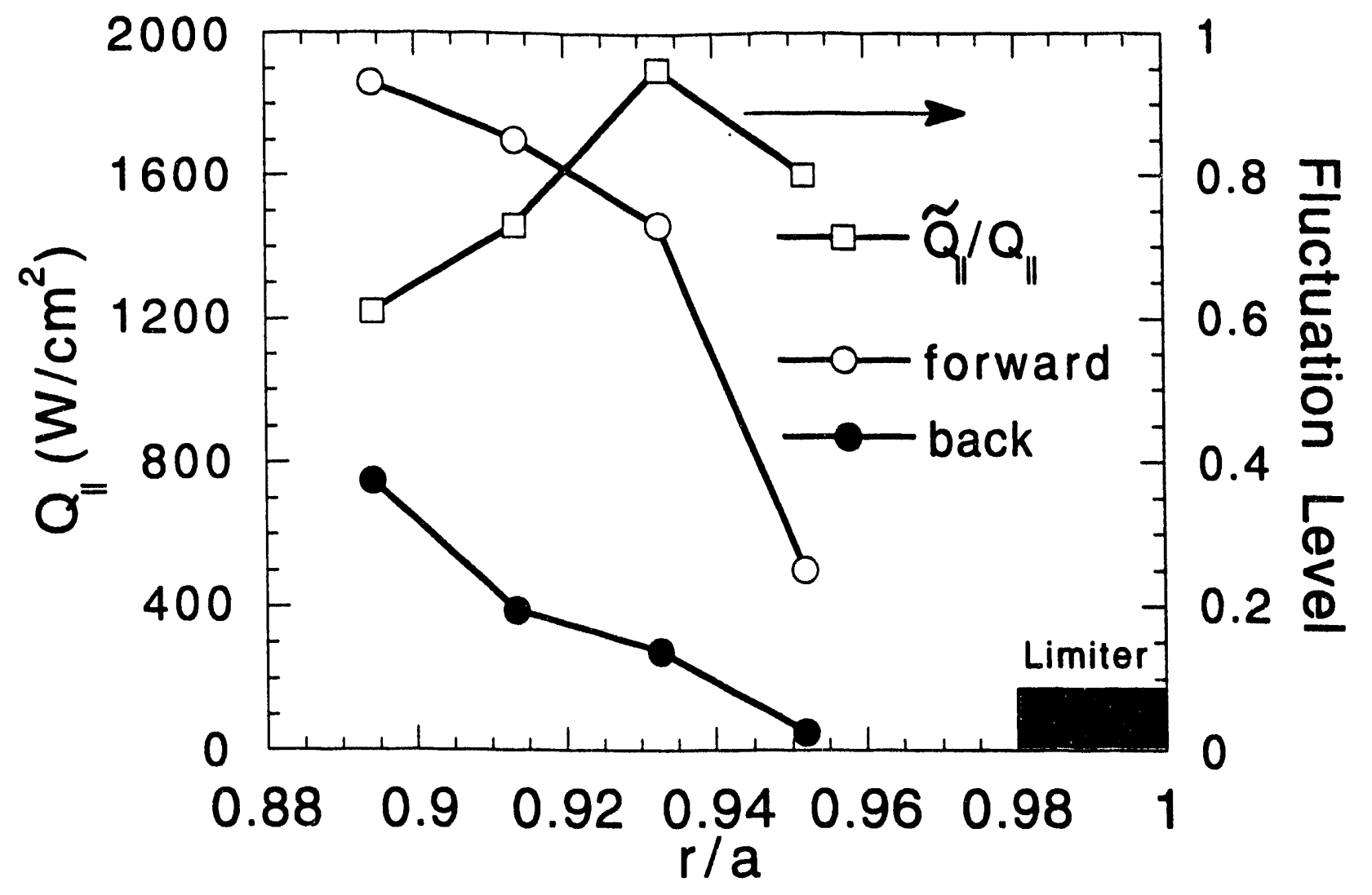

Fig. 3 

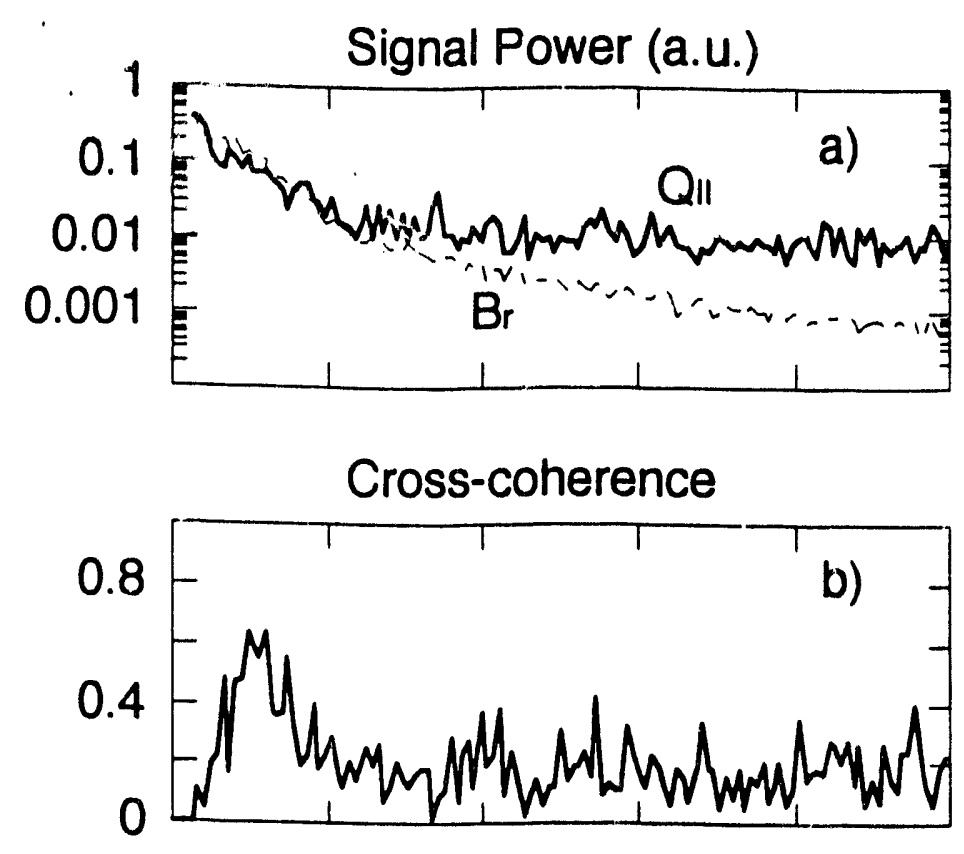

Cross-phase (deg)
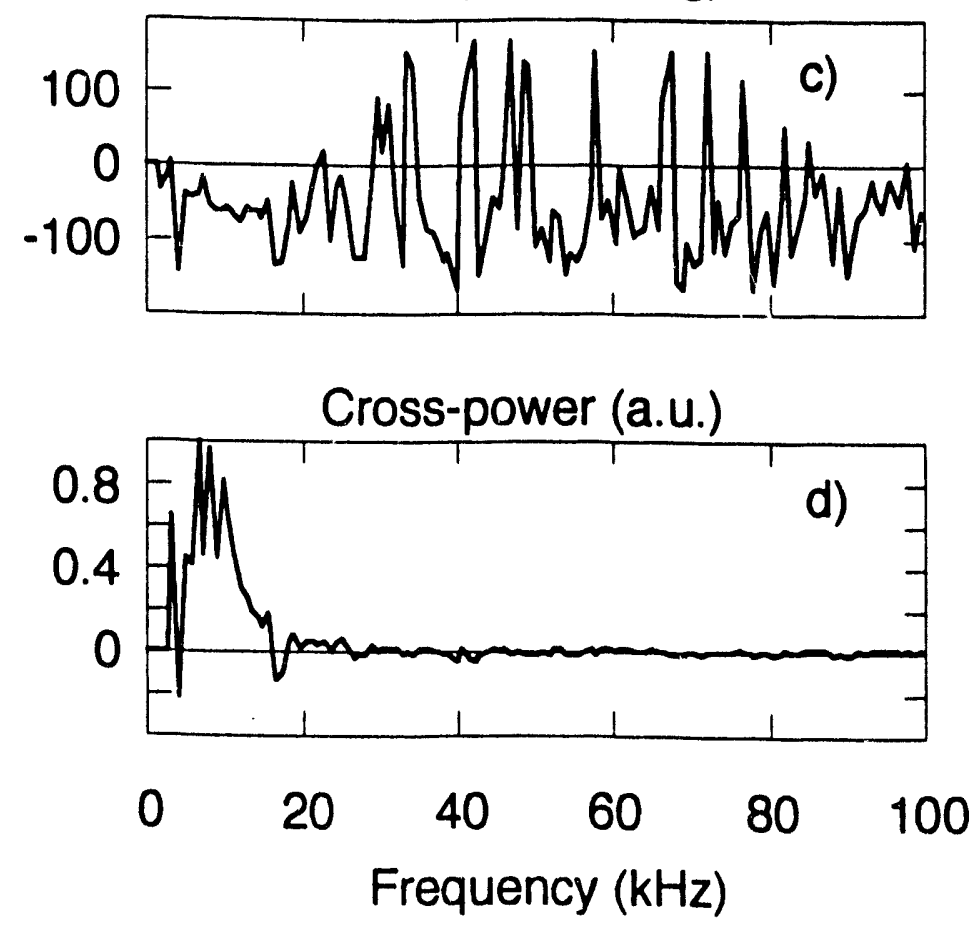

Fig. 4 


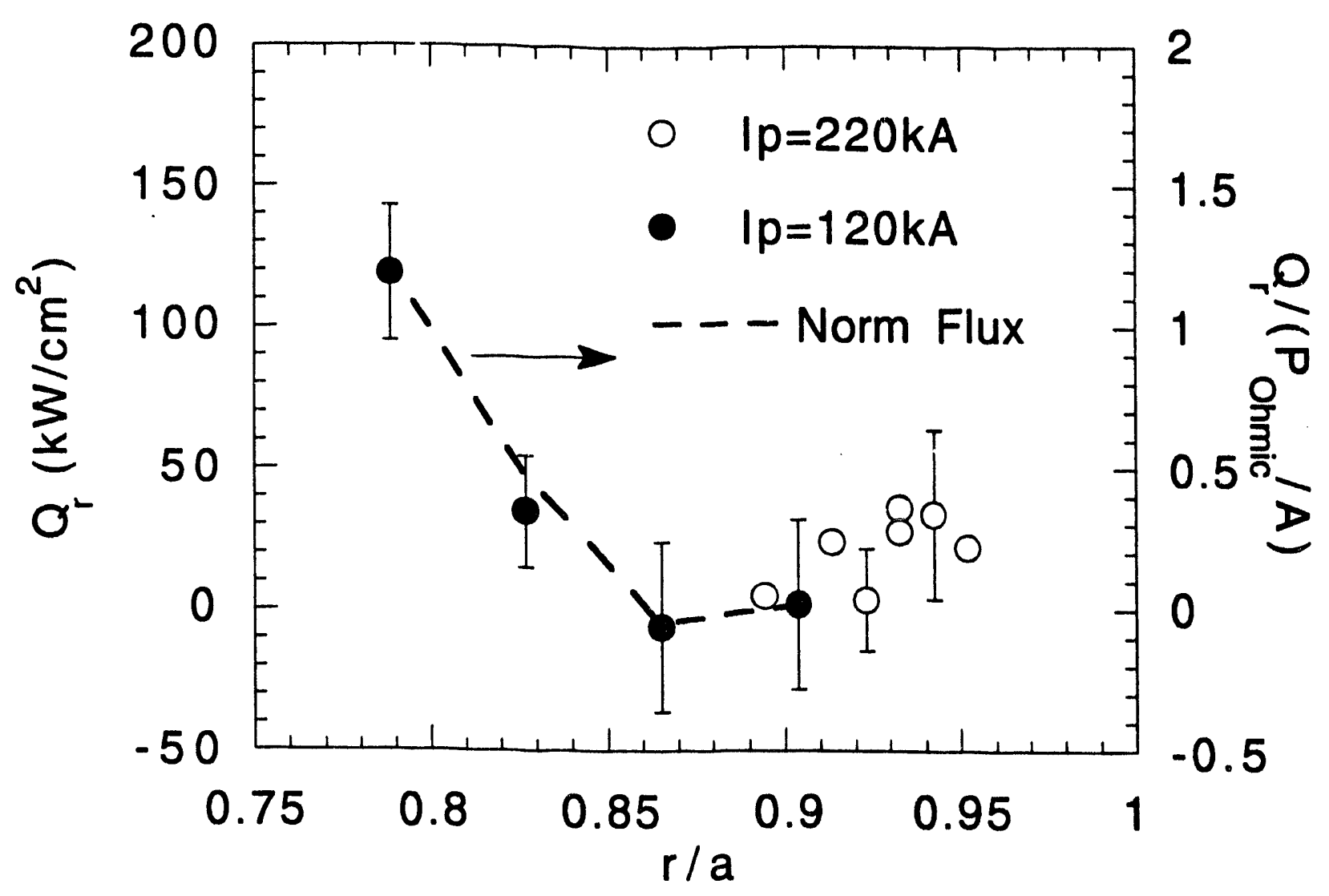

Fig. 5 
EXTERNAL DISTRIBUTION IN ADDITION TO UC-20

S.N. Rasband, Brigham Young University

T. Dolan, EG\&G Idaho, Inc.

R.A. Moyer, General Atornics

J.B. Taylor, Institute for Fusion Studies, The University of Texas at Austin

E. Uchimoto, University of Montana

F.W. Perkins, PPPL

O. Ishihara, Texas Technical University

M.A. Abdou, University of California, Los Angeles

R.W. Conn, University of California, Los Angeles

P.E. Vandenplas, Association Euratom-Etat Belge, Belgium

Centro Brasileiro de Pesquisas Firicas, Brazil

P. Sakanaka, Institute de Fisica-Unicamp, Brazil

Mme. Monique Bex, GANIL, France

J. Radet, CEN/CADARACHE, France

University of Ioannina, Greece

S. Ortolani, Istituto Gas Ionizzati, EURATON-ENEA-CNR Association, Italy

R. Andreani, Associazione EURATOM-ENEA sulla Fusione, Italy

Plasma section, Energy Fundamentals Division Electrotechnical Laboratory, Japan

Y. Kondoh, Gunma University, Kiryu, Gunma, Japan

H. Toyama, University of Tokyo, Japan

Z. Ypsjoda. University of Tokyo, Japan

FOM-Instituut voor Plassmafysica "Rijnhuizen," The Netherlands

Z. Ning, Academia Sinica, Peoples Republic of China

P. Yang, Shandong University, Peoples Republic of China

S. Zhu, University of Science \& Technology of China, People's Republic of China I.N. Bogatu, Institute of Atomic Physics, Romania

M.J. Alport, University of Natal, Durban, South Africa

R. Storer, The Flinders University of South Australia, South Australia

B. Lehnert, Royal Institute of Technology, Sweden

Librarian, CRPP, Ecole Polytechnique Federale de Lausanne, Switzerland

B. Alper, Culham Laboratory, UK

A. Newton, UK

2 for Chicago Operations Office

5 for individuals in Washington Offices

INTERNAL DISTRIBUTION IN ADDITION TO UC-20

80 for local group and file 

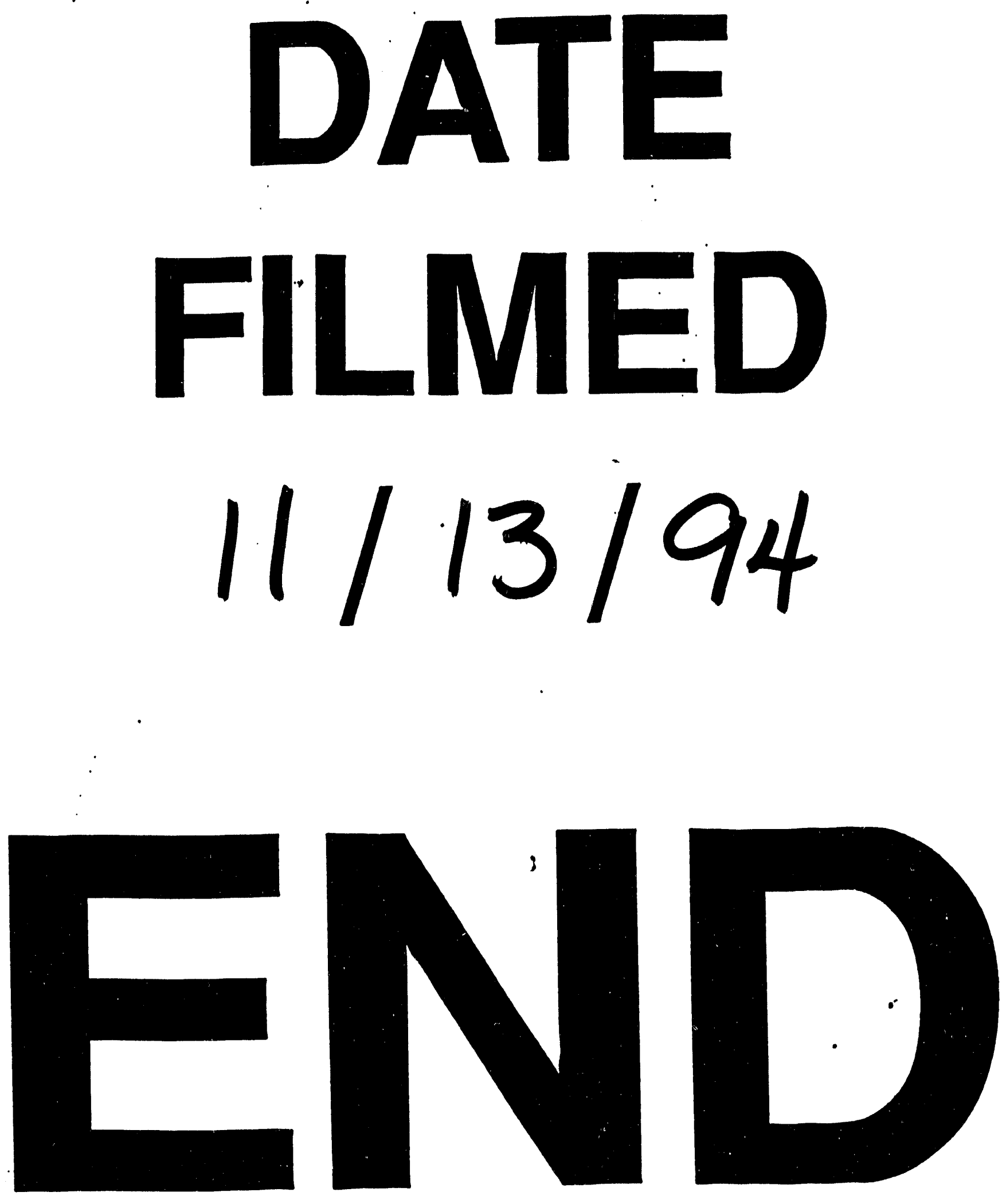
